\title{
Publisher's Note: Single-crystal growth and physical property characterization of the intermediate-valence compound $\mathrm{YbFe}_{2} \mathrm{Al}_{10}$ [Phys. Rev. B 95, 235132 (2017)]
}

\author{
J. L. Lv, R. Y. Chen, H. P. Wang, J. L. Luo, and N. L. Wang \\ (Received 19 June 2017; published 27 June 2017)
}

DOI: 10.1103/PhysRevB.95.239907

This paper was published online on 16 June 2017 with an incorrect figure. Figure 2 has been replaced as of 20 June 2017 . The figure is correct in the printed version of the journal. 